\title{
Estabilidade pós-contenção das alterações da forma do arco inferior na má oclusão de Classe II de Angle tratada com e sem a extração de pré-molares
}

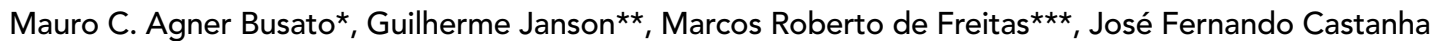 \\ Henriques ${ }^{\star \star \star \star}$
}

\section{Resumo}

Objetivo: o objetivo desse estudo foi comparar o comportamento da forma do arco inferior durante as fases de tratamento e pós-contenção, em pacientes portadores de má oclusão Classe II de Angle, que haviam se submetido ao tratamento ortodôntico com extrações de dois e de quatro pré-molares, portanto com e sem extrações no arco inferior. Metodologia: foram selecionados 66 pacientes que apresentavam inicialmente uma má oclusão de no mínimo meia Classe II, e que foram tratados com uma das combinações de extrações propostas. Esses pacientes foram divididos em dois grupos, sendo o grupo 1 composto de 19 pacientes tratados com extração de dois prémolares superiores, com idade média de 14,04 anos; e o grupo 2 composto de 47 pacientes com extração de quatro pré-molares, com idade média de 13,03 anos. Para verificação das diferenças entre os grupos, mediu-se os modelos referentes às fases pré-tratamento, pós-tratamento e, em média, cinco anos após o final do tratamento ativo. As alterações das dimensões do arco inferior (distância intercaninos, intermolares e comprimento de arco), entre as três fases, também foram comparadas. Resultados: os resultados demonstraram que diferenças estatisticamente significantes durante o tratamento se devem à opção por extrações no grupo 2. Não houve diferença entre os dois grupos para as mudanças pós-contenção no comprimento e na largura do arco inferior. Conclusões: pode-se esperar o mesmo grau de recidiva pós-contenção da forma do arco inferior em pacientes com má oclusão de Classe II, quando o tratamento é conduzido com extrações de dois pré-molares superiores, ou quando realizado com extrações de quatro pré-molares.

Palavras-chave: Estabilidade. Forma do arco inferior. Classe II de Angle. Extrações de pré-molares.

\section{INTRODUÇÃO}

A estabilidade do tratamento ortodôntico talvez seja, dentre as metas a serem alcançadas por esta terapia, a de mais difícil obtenção ${ }^{18,19}$. Angle ${ }^{1,2}$, em 1907, já afirmava que as recidivas devem ser esperadas, se os dentes não permanecerem,

* Professor de Ortodontia (graduação e especialização) Universidade Estadual do Oeste do Paraná (UNIOESTE). Especialista em Ortodontia pela EAPABO-Ponta Grossa-PR. Mestre em Ortodontia pela UEL-FOB/USP. Doutorando em Ortodontia pela FOA-UNESP-Araçatuba-SP.

** Professor Associado da Disciplina de Ortodontia da Faculdade de Odontologia de Bauru - USP.

*** Professor Associado de Ortodontia da Faculdade de Odontologia de Bauru da Universidade de São Paulo-FOB - USP.

**** Professor Titular do Departamento de Odontopediatria e Ortodontia e Saúde Coletiva da Faculdade de Odontologia de Bauru - USP. Coordenador dos Cursos de Pós-graduação em Ortodontia, ao nível de Doutorado e Especialização da Faculdade de Odontologia de Bauru. 
ao final do tratamento, em oclusão normal.

Está bem estabelecido na literatura que aumentar a largura e o comprimento do arco dentário, durante o tratamento, leva a uma tendência de retorno às dimensões iniciais, no período pós-contenção. Os dentes e as estruturas de suporte apresentam uma tendência natural de se moverem em direção à má oclusão inicial ${ }^{6,11,19,22,23,25,26,29}$.

A tendência dos dentes e estruturas de suporte de sofrerem alterações dimensionais durante o tratamento pode acarretar mudanças nas formas dos arcos dentários. Vários autores ${ }^{10,21,25}$ acreditam que o estabelecimento adequado da forma e da dimensão do arco dentário inferior é um fator de estabilidade dos resultados terapêuticos, e que a elaboração imprópria dos arcos pode contribuir com muitos efeitos indesejáveis, durante e após o tratamento. Outros autores ${ }^{29}$, porém, não correlacionaram o aumento das distâncias intercaninos, intermolares e do comprimento do arco, com a recidiva do apinhamento ântero-inferior. Muitos deles citaram que a alteração destes fatores pode sim ser estável e permanente ${ }^{29}$.

Strang ${ }^{25}$, em 1949, enfatizou que a expansão do arco dentário e o aumento da distância intercaninos podem ser causas da recidiva.

Little e Riedel ${ }^{18}$, em 1989, avaliaram casos 10 anos pós-contenção que apresentavam diastemas generalizados nos dentes anteriores antes do início do tratamento. Estes casos mostraram grande redução do comprimento do arco e da distância intercaninos, progressivamente com o tempo. $\mathrm{O}$ apinhamento pós-contenção foi mínimo na maioria dos casos, e os diastemas fechados não se abriram novamente. A hipótese de não se usar contenções ou que estas poderiam ser usadas por um tempo mínimo provou ser incorreta.

Em 1993, Rossouw et al..$^{21}$ avaliaram as alterações longitudinais de 88 pacientes tratados com mecânica Edgewise, 44\% sem e 56\% com extrações de pré-molares. As variáveis estudadas foram: trespasses vertical e horizontal, comprimento do arco inferior, posição cefalométrica dos incisivos superiores e inferiores, distância intercaninos e intermolares e o índice de irregularidade de Little. O comprimento do arco se achou diminuído e foi a única variável não estável na fase pós-contenção. Concluiu-se, então, que a expansão da distância intercaninos inferiores pode levar a uma falha nos resultados, e o aumento do comprimento do arco tem uma relação causal no apinhamento ânteroinferior pós-tratamento. Os resultados, quando comparados os grupos com e sem extrações, se mostraram semelhantes.

De la Cruz et al. ${ }^{10}$, em 1995, estudaram pacientes com más oclusões de Classe I e II de Angle, tratados com extrações de quatro pré-molares, e avaliaram as alterações longitudinais da forma do arco inferior até 10 anos pós-contenção. Notaram que, quanto maior a alteração durante o tratamento, maior será a recidiva. A forma do arco na fase pós-contenção tendeu a retornar às dimensões prévias ao tratamento.

Em 1995, Kahl-Nieke, Fischbach e Schwarze ${ }^{14}$ analisaram modelos de 226 pacientes com diversos tipos de más oclusões e observaram o comportamento das seguintes variáveis: distância intercaninos, intermolares, comprimento do arco, dimensão mésio-distal dos incisivos, índice de irregularidade de Little, apinhamento ântero-inferior, relação de caninos e molares e trespasses vertical e horizontal. Também relacionaram estas variáveis para verificar a influência do gênero, do alinhamento inicial e final dos incisivos, do tipo de mecânica utilizada, da quantidade de movimento dentário e da presença ou não de molares durante a fase pós-contenção. As variáveis pré-tratamento, como dimensão mésio-distal das coroas aumentada, apinhamento ântero-inferior severo, índice de irregularidade de Little alto, deficiência do comprimento do arco, constricção do mesmo, grande sobremordida, bem como o espaçamento pós-tratamento, expansão do arco, aumento do comprimento do arco e relações residuais de Classe II ou III de molar se mostraram fatores associados ao processo de aumento do apinhamento ântero-inferior 
e do índice de irregularidade pós-contenção. Dois terços da amostra apresentaram apinhamento ântero-inferior pós-contenção inceitável e quase $50 \%$ desta mostrou aumento no índice de irregularidade de Little. Os autores ${ }^{14}$ também encontraram que o subgrupo que sofreu extrações mostrou mais recidiva do apinhamento ântero-inferior e de rotações. As expansões exageradas do arco foram consideradas um fator de influência na recidiva, mas as extrações não preveniram esta recidiva.

Begole, Fox e Sadowsky ${ }^{4}$, em 1998, realizaram um estudo sobre as formas dos arcos. Os achados sugerem que a estabilidade pode não estar relacionada com a quantidade de alteração produzida durante o tratamento. Uma significante expansão pode ser conseguida nas regiões dos pré-molares, podendo-se esperar a sua estabilidade. O maior ganho na largura do arco foi para os segundos pré-molares; seguidos dos primeiros prémolares, molares e, então, os caninos. As distâncias intercaninos para ambos os arcos diminuíram em relação aos valores pré-tratamento. Essa estabilidade na região de pré-molares já havia sido evidenciada por Gardner e Chaconas ${ }^{11}$.

Burke et al. ${ }^{5}$, em 1998, pelo processo da metanálise de 26 artigos publicados e de algumas dissertações de Mestrado (não publicadas), avaliaram as alterações na largura intercaninos inferior após o período de contenção. A amostra combinada compreendeu 1.233 indivíduos, que foram divididos em subgrupos baseados na classificação da má oclusão e no tipo de tratamento em relação às extrações. Como conclusões, observaram que: a largura intercaninos tende a se expandir durante $o$ tratamento, entre 0,8 e $2 \mathrm{~mm}$, independentemente da classificação da má oclusão e do tratamento ter sido realizado com ou sem extrações; esta mesma largura tende a diminuir, após o período de contenção, entre 1,2 e 1,9mm, o que leva a uma alteração "líquida" no período pós-contenção da ordem de $0,5 \mathrm{~mm}$ de expansão e $0,6 \mathrm{~mm}$ de constricção. Já que a alteração "líquida" na largura intercaninos foi de aproximadamente zero, esse estudo claramente reforça o conceito da manutenção da largura intercaninos original no tratamento ortodôntico.

Para Toigo e Mandetta ${ }^{26}$, as maiores alterações na forma do arco no período pós-contenção ocorreram quando aconteceram grandes alterações com o tratamento. Segundo os autores, a manutenção da distância intercaninos durante o tratamento ortodôntico não tem influência no apinhamento dos incisivos inferiores, pois seus casos foram tratados sem expansão e, mesmo assim, apresentaram estreitamento e apinhamento no período pós-contenção ${ }^{26}$. O conceito de Strang ${ }^{25}$ e Shapiro ${ }^{22}$, de que a distância intercaninos deveria permanecer inalterada para se alcançar estabilidade, não se confirmou. Também não foi corroborada a idéia de Strang ${ }^{25}$ de que a extração de pré-molares permite o movimento distal dos caninos para uma área de maior distância óssea, o que proporcionaria uma estabilidade da expansão.

Com relação ao plano de tratamento para a má oclusão de Classe II de Angle, este, de uma maneira geral, pode ou não envolver a extração de dentes permanentes, dependendo das características inerentes ao caso ${ }^{3,7}$. No caso da opção por extrações, os pré-molares são os dentes preferencialmente eleitos, sendo que a combinação pode incluir as extrações de apenas dois pré-molares superiores $^{20}$ ou de quatro pré-molares ${ }^{3,7}$, ou seja, a Classe II de Angle pode ser tratada com ou sem extrações no arco inferior.

Este trabalho teve como objetivo verificar as diferenças no comportamento pós-contenção da forma do arco inferior, em uma amostra com má oclusão de Classe II tratada com extração de dois pré-molares superiores e outra amostra tratada com a extração de quatro pré-molares.

\section{MATERIAL E MÉTODOS Material}

A amostra foi obtida a partir dos arquivos da Disciplina de Ortodontia da Faculdade de Odontologia de Bauru - Universidade de São Paulo. 
Os pacientes apresentavam registros completos e foram selecionados de acordo com o critério de serem portadores de, no mínimo, uma má oclusão de meia Classe II de Angle, sendo que, de acordo com a severidade, os pacientes escolhidos mostravam, inicialmente, uma relação de meia Classe II até uma Classe II completa. Os pacientes deviam apresentar, ao início do tratamento, todos os dentes permanentes até os primeiros molares e ausência de anodontias e supranumerários, examinados na radiografia ortopantomográfica inicial.

A amostra foi dividida em dois grupos de acordo com a modalidade de tratamento da Classe II, ou seja, tratamentos com extração de dois pré-molares superiores (grupo 1) e tratamentos executados com a extração de quatro pré-molares (grupo 2). O grupo 1 foi composto por 19 pacientes, sendo 9 do gênero masculino e 10 do gênero feminino, com média de idade de 14,04 anos e desviopadrão de 1,74 anos. O tempo médio de duração do tratamento foi de 2,41 anos (d.p.= 0,88 anos), e o tempo médio de observação pós-tratamento foi de 5,17 anos (d.p.= 2,4 anos). O grupo 2 foi composto por 47 pacientes, sendo 20 do gênero masculino e 27 do gênero feminino, com média de idade de 13,03 anos e desvio-padrão de 1,76 anos. O tempo médio de duração do tratamento foi de 2,7 anos (d.p.: 0,7 anos) e o tempo médio de observação pós-tratamento foi de 5,91 anos (d.p.: 2,75 anos).

Todos os pacientes foram tratados com mecânica do arco de canto, sendo que as extrações foram executadas logo após a montagem da ancoragem, e o tratamento começou em seguida a este procedimento. Dez, dos dezenove pacientes do grupo 1 , foram submetidos à técnica de desgaste interproximal preconizada por Sheridan ${ }^{24}$, a fim de se obter espaço para a correção do apinhamento inferior. Após o término do tratamento corretivo, seguiu-se a fase de contenção, que consistiu no uso, para todos os pacientes, de uma barra lingual inferior 3x3 colada ou com bandas e contenção móvel tipo placa de Hawley no arco superior.
Estas contenções foram usadas seguindo-se o protocolo recomendado pela Disciplina de Ortodontia da FOB-USP, que é de seis meses com uso contínuo e mais seis meses de uso noturno para a placa de Hawley, e em média 2 anos para a contenção fixa $3 \times 3$ colada no arco inferior, sendo que estas contenções foram removidas na seqüência.

Os dados foram coletados a partir de três modelos inferiores dos pacientes, em períodos correspondentes ao início do tratamento, final do tratamento e período pós-tratamento médio de 5 anos, sendo este último relativo ao registro do modelo inferior sem a presença da barra lingual $(3 \times 3)$ colada. A qualidade dos resultados pós-tratamento e pós-contenção não foi considerada no processo de seleção da amostra. Na verdade todos os esforços foram executados para coletar registros e dados sem influências.

\section{Métodos}

Medições nos modelos de gesso

Foram coletadas as seguintes medidas nos três modelos inferiores de cada paciente:

- Largura intercaninos inferiores: distância em milímetros (medida com paquímetro) entre a ponta das cúspides dos caninos inferiores ou entre as pontas de cúspide estimadas, no caso de facetas de desgaste estarem presentes (Fig. 1).

- Comprimento do arco inferior: somatório da distância do ponto de contato anatômico mesial dos primeiros molares permanentes direito e esquerdo ao ponto de contato dos incisivos centrais (Fig. 1).

- Largura intermolares inferior: distância entre a ponta da cúspide mesiovestibular do $1^{\circ}$ molar inferior direito e a mesma referência anatômica do $1^{\circ}$ molar do lado oposto (Fig. 1).

\section{Análise estatística}

Erro do método

Para a avaliação do erro intra-examinador, foram realizadas novamente todas as medidas nos modelos de dezesseis pacientes de ambos os grupos, 
selecionados aleatoriamente, totalizando quarenta e oito modelos inferiores. A aplicação da fórmula proposta por Dahlberg ${ }^{9}\left(\mathrm{Se}^{2}=\right.$ somatória $\left.\mathrm{d}^{2} / 2 \mathrm{n}\right)$ permitiu estimar a ordem de grandeza dos erros casuais. A obtenção dos erros sistemáticos procedeu-se pela aplicação do teste t pareado, de acordo com Houston ${ }^{13}$.

\section{Método estatístico}

Para a análise estatística dos dados utilizou-se o teste $t$ para variáveis independentes para a comparação das medidas ao início, ao final do tratamento e cinco anos pós-tratamento, e para comparar as alterações ocorridas entre os três períodos avaliados, para os dois grupos. Os resultados foram considerados estatisticamente significantes para $\mathrm{p}<0,05$.

\section{RESULTADOS E DISCUSSÃO Precisão da metodologia}

Para avaliar os erros da metodologia, Houston ${ }^{13}$ recomenda, idealmente, que as medições sejam realizadas duas vezes. Entretanto, se isto não for possivel, o autor aconselha que os modelos sejam selecionados ao acaso, da amostra total. Neste estudo, foram medidos novamente dezesseis mode-

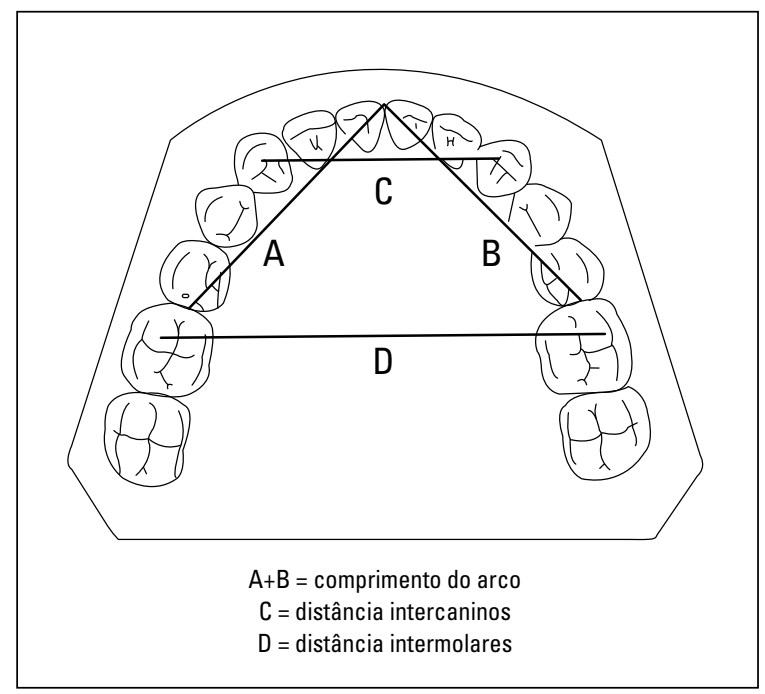

FIGURA 1 - Técnica de Little ${ }^{20}$ para medição dos modelos. los inferiores, escolhidos ao acaso, número esse julgado suficiente, devido à quantidade de variáveis utilizadas e com base em estudos similares.

A maior fonte de erros casuais, segundo Hous$\operatorname{ton}^{13}$, acontece pela dificuldade de identificação de um ponto em particular ou pela imprecisão na definição de certos pontos. De uma forma geral, os erros casuais nesse trabalho foram bastante reduzidos, exceto para o comprimento do arco inicial, que foi de 2,43mm (Tab. 1). Como os erros casuais para as variáveis em estudo foram bastante reduzidos, conclui-se que os desvios-padrão encontrados para elas sejam realmente o reflexo da variabilidade dos grupos.

No entanto, não houve diferenças estatisticamente significantes na avaliação do erro sistemático $(\mathrm{p}<0,05)$, em nenhuma das variáveis avaliadas. Os erros sistemáticos se manifestam quando um determinado parâmetro é persistentemente sub ou superestimado, podendo ocorrer quando um pesquisador modifica a sua técnica de mensuração após certo tempo ou então quando ele tende, subconscientemente, a direcionar os resultados de acordo com as suas expectativas em relação às conclusões do estudo ${ }^{13}$.

\section{Alterações da largura intercaninos durante e após o tratamento ortodôntico}

Analisando-se os resultados obtidos, verificouse uma diferença estatisticamente significante entre os grupos tratados com a extração de apenas dois pré-molares superiores (grupo 1) e o grupo tratado com a extração de quatro pré-molares (grupo 2), com relação ao comportamento para a distância intercaninos durante a terapia ortodôntica, com uma diminuição desta dimensão no grupo $1(-0,27 \mathrm{~mm})$ e um aumento na amostra tratada com extração $(1,87 \mathrm{~mm})$, como mostra a tabela 2 . Isso demonstra que precauções foram tomadas no grupo sem extrações no arco inferior, no sentido de se evitar, durante o tratamento, a expansão transversal dos caninos, visto que, neste grupo, dez dos dezenove pacientes foram submetidos a des- 
Tabela 1 - Apresentação dos erros casuais (Dahlberg) e dos resultados do teste t para amostras dependentes, do teste intraexaminador.

\begin{tabular}{|c|c|c|c|c|c|c|}
\hline \multirow{2}{*}{ variáveis } & \multicolumn{2}{|c|}{$1^{\mathrm{a}}$ medição } & \multicolumn{2}{|c|}{$2^{\mathrm{a}}$ medição } & \multirow{2}{*}{ Dahlberg } & \multirow{2}{*}{ p } \\
\hline & média & d.p. & média & d.p. & & \\
\hline $3 \times 3(1)$ & 25,37 & 1,32 & 25,45 & 1,09 & 0,06 & 0,41 \\
\hline $3 \times 3(F)$ & 25,84 & 2,03 & 25,89 & 1,86 & 0,06 & 0,59 \\
\hline $3 \times 3(P C)$ & 24,78 & 2,11 & 24,78 & 1,95 & 0,03 & 0,95 \\
\hline $6 \times 6$ (I) & 36,69 & 2,49 & 36,84 & 2,44 & 0,04 & 0,06 \\
\hline $6 \times 6(F)$ & 36,20 & 2,31 & 36,39 & 2,27 & 0,09 & 0,08 \\
\hline $6 \times 6(P C)$ & 36,01 & 2,75 & 35,96 & 2,57 & 0,11 & 0,73 \\
\hline comp. arco (I) & 56,11 & 3,48 & 54,64 & 7,72 & 2,43 & 0,38 \\
\hline comp. $\operatorname{arco}(\mathrm{F})$ & 51,20 & 5,2 & 51,22 & 4,97 & 1,16 & 0,94 \\
\hline comp. $\operatorname{arco}(\mathrm{P})$ & 49,24 & 5,36 & 49,71 & 5,19 & 1,52 & 0,31 \\
\hline
\end{tabular}

* Estatisticamente significante $(p<0,05)$.

Tabela 2 - Resultado do teste t independente, entre os grupos 1 e 2.

\begin{tabular}{|c|c|c|c|c|c|c|}
\hline \multirow{2}{*}{ variáveis } & \multicolumn{2}{|c|}{ grupo 1} & \multicolumn{2}{|c|}{ grupo 2} & \multirow{2}{*}{ gl } & \multirow{2}{*}{$\mathbf{p}$} \\
\hline & média & d.p. & média & d.p. & & \\
\hline idade & 14,04 & 1,74 & 13,03 & 1,76 & 64 & $0,04^{*}$ \\
\hline duração do tratamento & 2,41 & 0,88 & 2,70 & 0,70 & 64 & 0,16 \\
\hline controle pós-tratamento & 5,17 & 2,40 & 5,91 & 2,75 & 64 & 0,31 \\
\hline $3 \times 3(1)$ & 25,67 & 1,76 & 25,70 & 2,04 & 64 & 0,96 \\
\hline $3 \times 3(F)$ & 25,41 & 1,92 & 26,62 & 1,38 & 64 & $0^{*}$ \\
\hline $3 \times 3(P C)$ & 24,71 & 1,76 & 25,67 & 1,55 & 64 & $0,03^{*}$ \\
\hline $6 \times 6$ (I) & 39,82 & 2,98 & 38,55 & 2,31 & 64 & 0,06 \\
\hline $6 \times 6(F)$ & 39,14 & 2,51 & 36,40 & 1,98 & 64 & $0^{*}$ \\
\hline $6 \times 6(P C)$ & 39,26 & 3,20 & 36,51 & 2,17 & 64 & $0^{*}$ \\
\hline comp. arco (I) & 59,20 & 4,17 & 57,87 & 3,83 & 64 & 0,21 \\
\hline comp. arco (F) & 59,21 & 4,24 & 49,42 & 2,99 & 64 & $0^{*}$ \\
\hline comp. $\operatorname{arco}(\mathrm{P})$ & 57,95 & 3,99 & 47,81 & 2,92 & 64 & $0^{*}$ \\
\hline
\end{tabular}

${ }^{*}$ Estatisticamente significante $(p<0,05)$.

gaste interproximal na região de incisivos inferiores, para acomodar o apinhamento ântero-inferior prévio ao tratamento. Já o aumento na largura intercaninos para o grupo é explicado pelo posicionamento distal dos caninos inferiores, ocupando o espaço da extração e conseqüentemente aumentando esta dimensão do arco inferior ${ }^{27}$.

A largura intercaninos não mostrou diferença estatisticamente significante entre os grupos, quando se considerou o período pós-contenção, sendo que esta variável diminuiu no grupo 1 (-0,69mm) e no grupo $2(-0,95 \mathrm{~mm})$, confirmando os achados na literatura da tendência de constrição desta medida na fase de pós-contenção, perdendo-se a expansão conseguida durante o tratamento, como resultado da recidiva dos caninos em direção à sua posição original, independente se o tratamento foi executado com ou sem extrações de pré-molares no arco inferior ${ }^{19,28}$. Gardner e Chaconas ${ }^{11}$ encontraram uma maior constrição pós-contenção na largura intercaninos para o grupo tratado com extrações. 
Little et al. ${ }^{19}$ afirmaram que esta constrição da largura intercaninos é progressiva, principalmente na primeira década pós-tratamento. $\mathrm{Na}$ segunda década pós-contenção, segundo os autores ${ }^{19}$, a constrição continua, porém em menor grau.

\section{Alterações da largura intermolares durante e após o tratamento ortodôntico}

Os resultados mostram que, durante o tratamento, houve uma diminuição da distância intermolares para os dois grupos, sendo de $0,68 \mathrm{~mm}$ para o grupo 1 e de $2,15 \mathrm{~mm}$ para o grupo 2 , mostrando uma diminuição significativamente maior do ponto de vista estatístico, no grupo com quatro extrações de pré-molares (Tab. 2). Isto se deve à mesialização dos molares para regiões mais estreitas do arco dentário, durante o fechamento dos espaços das extrações inferiores no grupo 2 .

No entanto, durante a fase de pós-contenção, houve um pequeno aumento na largura intermolares, sendo que este aumento se mostrou similar para os dois grupos. Esta semelhança de comportamento dos grupos tratados com e sem extrações no arco inferior na fase de pós-contenção, para a largura intermolares, também foi um achado de outros trabalhos ${ }^{15,28}$, embora estes trabalhos mostrem uma diminuição desta dimensão no período pós-contenção. Uhde et al. ${ }^{28}$ relacionaram esta diminuição pós-contenção da distância intermolares, com o aumento desta distância durante o tratamento.

Alguns trabalhos mostram comportamentos diferentes quando se comparam casos tratados com e sem extrações, e quando se leva em conta a estabilidade em longo prazo da largura intermolares $^{8,29}$. Nos casos com extrações, a distância intermolares diminui durante o tratamento e continua a decrescer na pós-contenção; nos casos sem extrações, houve uma manutenção desta distância durante o tratamento e em alguns casos aumento na distância intermolares na fase de pós-contenção $0^{8,29}$.

Para Uhde et al. ${ }^{28}$, as poucas mudanças que foram observadas durante e após o tratamento, tendem a sustentar o conceito da estabilidade da distância intermolares, em especial nos casos de Classe II, os quais mantiveram pequenos aumentos na largura intermolares, no período pós-contenção ${ }^{29}$.

\section{Alterações do comprimento do arco durante e após o tratamento ortodôntico}

As diferenças no comprimento do arco, nos grupos com e sem extrações, durante o tratamento, são facilmente explicadas, segundo Shapiro ${ }^{22}$. Se dentes são extraídos, espera-se que o comprimento do arco diminua de forma significativa. $\mathrm{Na}$ amostra com quatro extrações (grupo 2) houve uma diminuição de $8,45 \mathrm{~mm}$ e na amostra com extrações de apenas dois pré-molares (grupo 1), ocorreu a manutenção do comprimento do arco durante o tratamento (Tab. 2).

Na pós-contenção, os dois grupos não mostraram diferenças na diminuição do comprimento do arco, corroborando os achados de Little et al. ${ }^{19}$; Gardner e Chaconas ${ }^{11}$, que afirmaram que: "um fator que se mostra consistentemente previsivel é a diminuição contínua do comprimento do arco mandibular, que ocorre com o tempo, após a remoção das contenções". Para o grupo 1 a diminuição pós-contenção do comprimento do arco foi de $1,26 \mathrm{~mm}$ e para o grupo 2 foi de $1,60 \mathrm{~mm}$, diferença esta que não apresenta relevância do ponto de vista estatístico (Tab. 2).

As reduções do comprimento de arco encontradas neste trabalho são um pouco menores do que nos casos tratados com extrações e 10 anos sem contenção ${ }^{16}(-2,5 \mathrm{~mm})$, porém deve-se considerar que o tempo pós-contenção das amostras de Classe II, tratadas com a extração de dois e quatro pré-molares, é a metade do utilizado no trabalho referido, e portanto, com o aumento do tempo de controle destas amostras, espera-se uma maior diminuição no comprimento do arco, principalmente como reflexo do processo de maturação oclusal, que atua sobre esta dimensão ${ }^{17}$. 


\section{CONCLUSÕES}

As alterações pós-contenção na forma do arco inferior no grupo de Classe II tratado com extrações de dois pré-molares superiores foram semelhantes, do ponto de vista estatístico, às do grupo de Classe II, tratado com extrações de quatro prémolares.

\title{
Postretention lower arch form stability in Angle Class II patients treated with and without premolars extractions
}

\begin{abstract}
Aim: the purpose of this research was to compare the relapse of the postretention lower arch form in patients with Class II malocclusions, that were treated with either two or four premolar extraction therapies. Methods: Group 1 consisted of 19 patients ( 9 male e 10 female) treated with two premolar extraction, and group 2 consisted of 47 patients ( 20 male e 27 female) treated with four premolar extraction. The initial mean age of the first group was 14.04 years, and the initial mean age for group two was 13.03 years. Individual variables of the mandibular arch, such as intercanine width, intermolar width and arch length, were evaluated in the pre-treatment, end of active treatment and five years posttreatment diagnostic casts of each patient. Independent $t$ tests were used to compare the differences of the variables between groups. Results: results demonstrated that there were no statistically significant difference between the groups for posttreatment constriction of the mandibular arch (width and length). Conclusion: it was concluded that the treatment of Class II malocclusion with two premolar extraction provides the same stability for lower arch form as the treatment with four premolar extraction.
\end{abstract}

Key words: Stability. Lower arch form. Angle Class II malocclusion. Premolar extraction.

\section{REFERÊNCIAS}

1. ANGLE, E. H. The latest and best in orthodontic mechanism. Dent Cosmos, Philadelphia, v. 70, no. 12, p. 1143-1145, Dec. 1928.

2. ANGLE, E. H. Treatment of malocclusion of the teeth. Angle's System. Philadelphia: S. S. White, 1907. p. 479-489; 502-505; 514-517.

3. ARTUN J.; GAROL, J. D.; LITTLE, R. M. Long-term stability of mandibular incisors following sucessful treatment of Class II, division 1, malocclusions. Angle Orthod, Appleton, v. 66, no. 3, p. 229-238, 1996.

4. BEGOLE, E. A.; FOX, D. L.; SADOWSKY, C. Analysis of change in arch form with premolar expansion. Am J Orthod Dentofacial Orthop, St. Louis, v. 113, no. 3, p. 307-315, Mar. 1998.

5. BURKE, S. P.; SILVEIRA, A. M.; GOLDSMITH, J.; YANCEY, J. M.; STEWART, A. V.; SCARFE, W. C. A meta-analysis of mandibular intercanine width in treatment and postretention. Angle Orthod, Appleton, v. 68 , no. 1 , p. 53-60, 1998.

6. BISHARA, S. E.; JAKOBSEN, J. R.; TREDER, J. E.; STASI, M. J. Changes in the maxillary and mandibular tooth size-arch length relationship from early adolescence to early adulthood. Am J Orthod Dentofacial Orthop, St. Louis, v. 95, no. 1, p. 46-59, Jan. 1989.

7. BISHARA, S. E.; BAYATI, P.; ZAHER, A. R.; JAKOBSEN, J. R. Comparison of the dental arch changes in patients with Class II, division 1 malocclusions: extraction vs nonextraction treatments. Angle Orthod, Appleton, v. 64, no. 5, p. 351-358, 1994.

8. CELENZA, F.; HOCHMAN, M. N. Absolute anchorage in orthodontics: direct and indirect implant-assisted modalities. J Clin Orthod, Boulder, v. 34, no. 7, p. 397-402, July 2000.

9. DAHLBERG, G. Statistical methods for medical and biological students. New York: Interscience, 1940.
10. DE LA CRUZ, A.; SAMPSON, P.; LITTLE, R. M.; ARTUN, J.; SHAPIRO, P. A. Long-term changes in arch form after orthodontic treatment and retention. Am J Orthod Dentofacial Orthop, St. Louis, v. 107, no. 5, p. 518-530, May 1995.

11. GARDNER, S. D.; CHACONAS, S. J. Posttreatment and postretention changes following orthodontic therapy. Angle Orthod, Appleton, v. 46, no. 2, p.151-161, Apr. 1976.

12. GRABER, T. M. Current orthodontic concepts and techniques. Philadelphia: W. B. Saunders, 1975. v. 1.

13. HOUSTON, W. J. B. Analysis of errors in orthodontic measurements. Am J Orthod, St. Louis, v. 83, no. 5, p. 382-390, May 1983.

14. KAHL-NIEKE, B.; FISCHBACH, H.; SCHWARZE, C. W. Post-retention crowding and incisor irregularity: a long-term follow-up evaluation of stability and relapse. Br J Orthod, London, v. 22, no. 3, p. 249-257, Aug. 1995.

15. LITT, et al. Class II division 2 malocclusion: to extract or not extract? Angle Orthod, Appleton, v. 54, no. 2, p. 123-138, Apr. 1984.

16. LITTLE, R. M. The irregularity index: a quantitative score of mandibular anterior alignment. Am J Orthod, St. Louis, v. 68, no. 5, p. 554-563, Nov. 1975

17. LITTLE, R. M.; RIEDEL, R. A.; ARTUN, J. Changes in mandibular anterior alignment 10 to 20 years postretention. Am J Orthod Dentofacial Orthop, St. Louis, v. 93, no. 5, p. 423-428, May 1988.

18. LITTLE, R. M.; RIEDEL, R. A. Postretention evaluation of stability and relapse - mandibular arches with generalized spacing. Am J Orthod Dentofacial Orthop, St. Louis, v. 95, no. 1, p. 37-41, Jan. 1989.

19. LITTLE, R. M.; RIEDEL, R. A.; STEIN, A. Mandibular arch length increase during the mixed dentition: postretention evaluation of stability and relapse. Am J Orthod Dentofacial Orthop, St. Louis, v. 97, no. 5, p. 393-404, May 1990. 
20. OLIVEIRA JÚNIOR, G. et al. Estudo da recidiva do apinhamento ântero-inferior de casos tratados com extrações de quatro primeiros pré-molares e mecânica do arco de canto. Ortodontia São Paulo, v. 24, n. 1, p. 24-30, jan./abr. 1991.

21. ROSSOUW, P. E.; PRESTON, C. B.; LOMBARD, C. J.; TRUTER, J. W. A longitudinal evaluation of the anterior border of dentition. Am J Orthod Dentofacial Orthop, St. Louis, v. 104, no. 2, p. 146-152, Aug. 1993.

22. SHAPIRO, P. A. Mandibular dental arch form and dimension treatment and postretention changes. Am J Orthod, St. Louis, v. 66, no. 1, p. 58-70, July 1974.

23. SHIELDS, T.; LITTLE, R.; CHAPKO, M. Stability and relapse of mandibular anterior alignment: a cephalometric appraisal of first premolar extraction cases treated by traditional edgewise orthodontics. Am J Orthod, St. Louis, v. 87, no. 1, p. 27-38, Jan. 1985.

24. SHERIDAN, J. J. Air-rotor stripping update. J Clin Orthod, Boulder, v. 21, no. 11, p. 781-788, Nov. 1987.

25. STRANG, R. H. W. The fallacy of denture expansion as a treat- ment procedure. Angle Orthod, Appleton, v. 19, no. 1, p. 12-22, Jan. 1949

26. TOIGO, E.; MANDETTA, S. Estudo das alterações das dimensões e formas dos arcos dentários, ocorridas no final e cinco anos pós-tratamento, em pacientes com má oclusão de Classe II, divisão 1, tratados com extrações de pré-molares pela técnica de arco de canto. Ortodontia, São Paulo, v. 33, n. 2, p. 20-35, maio/ago. 2000.

27. TWEED, C. H. Indication for extraction of teeth in orthodontic procedures. Postretention study: incidence and stability of rotated teeth in humans. Am J Orthod Oral Surg, Chicago, v. 30, p. 405-428, 1944.

28. UHDE, M. D.; SADOWSKY, C.; BEGOLE, E. A. Long-term stability of dental relationships after orthodontic treatment. Angle Orthod, Appleton, v. 53, no. 3, p. 240-252, July 1983.

29. WALTER, D. C. Changes in the form and dimensions of dental arches resulting from orthodontic treatment. Angle Orthod, Appleton, v. 23, no. 1, p. 3-18, 1953.
Endereço de correspondência

Mauro C. Agner Busato

Av. Brasil, $7174,1^{\circ}$ andar, Sala 1 - Cascavel/PR

CEP: 85.806-000

E-mail: mcabusato@uol.com.br 